\title{
Continuous Gulture of Rumen Bacteria: Apparatus
}

\author{
By P. N. HOBSON \\ Rowett Research Institute, Bucksburn, Aberdeen
}

(Received 10 March 1964)

\begin{abstract}
SUMMARY
A small-scale apparatus for the continuous culture of anaerobic bacteria is described.
\end{abstract}

\section{INTRODUCTION}

The continuous culture of micro-organisms has been studied for a number of years and the most successful designs of apparatus have been based on the 'chemostat' of Monod (1950) and Novick \& Szilard (1950), and the 'turbidostat' of Fox \& Szilard (1955). As Herbert (1958) pointed out, the chemostat and turbidostat are essentially the same apparatus but they work best over different ranges of growth rate. The chemostat has some advantages over the turbidostat mechanically and for growth at low growth rates, and a chemostat type of apparatus was chosen for our work. Most of the chemostats described have been for the growth of aerobic bacteria and a problem in these designs is to supply sufficient air to the microorganisms, and this has led to the design of apparatus in which a film of culture flows around the walls of a vessel (e.g. Monod, 1950, and others; Dawson, 1963). This type of apparatus is not so suitable for anaerobic work as the 'stirred fermenter' type. The most important rumen bacteria are strict anaerobes which require special techniques for handling and are unable to initiate growth unless the media used are highly reduced. The apparatus described here was developed after initial experiments some 5 or 6 years ago when a pH-controlled batch culture apparatus was built in which large amounts of Streptococcus bovis were grown for the isolation of a polysaccharide. The merits of continuous culture for providing large amounts of micro-organisms for enzyme work and for analysis then became apparent. The rumen may be likened to a kind of continuous culture and it seemed that a better understanding of the behaviour of rumen bacteria might be obtained if they were grown in continuous, rather than batch, culture. The development of a comparatively large apparatus with an external culture circuit in which was incorporated the $\mathrm{pH}$ controls proved unsuccessful for strict anaerobes, partly because of the long lengths of silicone tubing involved in the peristaltic pumps. Sufficient air to oxidize the medium can diffuse through silicone tubing. The apparatus was then redesigned on a smaller scale for growth of strict anaerobes. The mechanically stirred fermenter designs such as those of Elsworth, Meakin, Pirt \& Capell (1956) were not followed because of expense and the difficulties of sealing the stirrer bearings, and because the bacteria which it was proposed to grow showed signs of being rather fragile and it was felt that a rapidly revolving stirrer might damage the organisms. A magnetic stirrer would overcome the difficulties of sealing bearings, but would still be liable to cause mechanical damage to the organisms. As a 
beginning the $\mathrm{pH}$ control was left out, the buffering action of the medium being relied on for $\mathrm{pH}$ control, but it has been found possible to incorporate in a larger vessel $(250 \mathrm{ml}$.) an internal electrode system operating, through a $\mathrm{pH}$ meter and relay, solenoid-operated alkali and acid inlets. Since experiments at low growth rates were planned to involve doubling times of 7 or $8 \mathrm{hr}$ or more, it was felt that the apparatus must be capable of running for much more than a day or two; and if it were to supply organisms for enzyme work it should run more or less indefinitely with minimum attention. The simplest design was used and all the parts of the present apparatus have been found necessary for its efficient functioning. The apparatus was demonstrated to the Physiological Society (Hobson \& Smith, 1962) and a brief description was given in 1963 (Hobson \& Smith, 1963).

\section{APPARATUS AND METHODS}

The apparatus (shown diagrammatically in Fig. 1) consists of the following parts. All joints are B5 ground glass joints. Rubber tubing is heavy wall red rubber of internal diameter approx. $\frac{1}{8}$ in. All rubber to glass joints are taped and wired on. $\mathrm{A}$ is a plastic balloon which holds about $5 \mathrm{l}$. of $\mathrm{CO}_{2}$ under atmospheric pressure. This is attached to the $5 \mathrm{l}$. flask $\mathrm{B}$ through a cottonwool filter and replaces the medium from flask $\mathrm{B}$ with $\mathrm{CO}_{2}$. When the $\mathrm{CO}_{2}$ in $\mathrm{A}$ is at more than atmospheric pressure it forces medium through the pump valves. Flask $\mathrm{B}$ is joined by rubber tubing and glass joints to a 2 l. conical flask $\mathrm{C}$ which is in a water bath at $39^{\circ}$. Flask $\mathrm{C}$ is then joined through a $\frac{1}{4}$ in. rubber tubing to the inlet pump $\mathrm{D}$. The distance between $\mathrm{C}$ and $\mathbf{D}$ is made as short as possible. The main purpose of heating flask $\mathbf{C}$ is to prevent bubble formation as the $\mathrm{CO}_{2}$-saturated medium is drawn up into the pump. Small bubbles stick to the ball valves of these pumps and stop medium flow at low pumping speeds. The inlet tube between $\mathrm{D}$ and $\mathrm{F}$ contains a gas-gap (E) which prevents back-growth of the culture up the inlet tube and into C. As this gap tends to fill with medium during long runs it was found most convenient to allow a very small stream of $\mathrm{CO}_{2}$, controlled by a fine jet and screw clips on the tubing $\mathrm{M}$, to pass through a filter into the side arm of E. Passage of a rapid flow of $\mathrm{CO}_{2}$ in with the medium tends to suck medium through the pumps. $F$ is the culture vessel, of working volume $120 \mathrm{ml}$., and it is fitted with a heavy rubber bung through which pass the tubes shown and an inoculation port, a B 5 cone and socket, G. The vessel is in a water bath at $39^{\circ} . \mathrm{H}$ is a subsidiary pump circuit, helping to stir the culture, but also acting as a sampling point through the two-way tap fitted with a hooded outlet tube. The main stream of $\mathrm{CO}_{2}$ passes from the $50 \mathrm{lb}$. cylinder $\mathrm{Q}$ through a reduction valve, a 'Rotameter' flow meter $\mathrm{P}$, a furnace containing red hot copper turnings $(\mathrm{O})$, through the branch point $\mathrm{N}$ and cottonwool filters to the culture vessel. The tubing of the $\mathrm{CO}_{2}$ line is pressure tubing which allows a negligible amount of air to permeate through, except for the short branch $\mathrm{M}$ which is $\frac{1}{8}$ in. tubing. The $\mathrm{CO}_{2}$ escapes from the culture vessel by the overflow tubing and carries excess medium with it into the 2 l. flask $I$ which is immersed in a refrigerated tank at $1^{\circ}$. I is fitted with a bunsen valve and cottonwool filter $(J)$ through which excess $\mathrm{CO}_{2}$ escapes. The outlet tube $\mathrm{K}$ is fitted with a tap and ground glass joint to which can be attached the flask $\mathbf{L}$ fitted with a cottonwool filter and a glass tube which can be attached to a water vacuum pump. Screw clips are attached at various points in the circuit. 
Between experiments the rubber tubing and cottonwool filters are usually replaced and the whole apparatus thoroughly cleaned. The culture vessel head with inlet pump circuit is separately wrapped, as are the circulating pump circuit, the flasks I and L, and the tops of the various flasks. All the separate pieces of apparatus are autoclaved at $120^{\circ}$ for $25 \mathrm{~min}$. to ensure thorough heating of pumps, etc. The various joint ends are separately covered under the main wrappings. The sterile apparatus is rapidly assembled as far as the joint below the pump $D$, the pumps are connected to the motors and connexions made from the $\mathrm{CO}_{2}$ lines to the filter inlets. The medium is separately prepared in flasks $\mathrm{C}$ and $\mathrm{B}$. The basal medium, without bicarbonate, cysteine and carbohydrate, is autoclaved in the flasks, which are

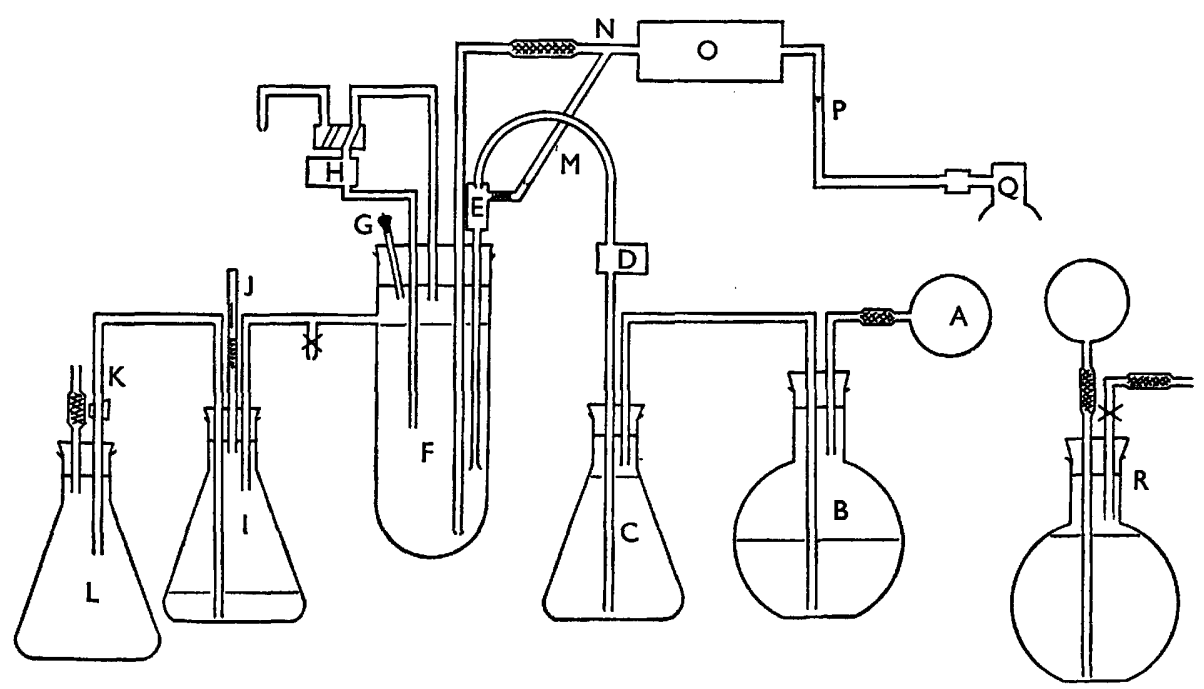

Fig. 1. Continuous culture apparatus. Diagrammatic. The lettered parts are referred to in the text. The hatched areas are cottonwool filters.

closed with aluminium foil, for $25 \mathrm{~min}$. at $120^{\circ}$. On removal from the autoclave, whilst the medium is still hot, the aluminium foil caps are replaced by the bungs carrying the inlet tubes, etc., but with a rubber bladder (a football bladder) of pure $\mathrm{CO}_{2}$ attached through a filter to the medium outlet tube, as in $\mathrm{R}$. The other tube with the filter is clipped off. Before the bungs are pressed home a strong stream of $\mathrm{CO}_{2}$ is bubbled through the medium to displace air in the flasks. When the medium has cooled a little, to say $60^{\circ}$, the bung is displaced and a concentrated solution of bicarbonate + cysteine + carbohydrate (filter sterilized) rapidly poured in whilst $\mathrm{CO}_{2}$ is allowed to escape around the bung. The bungs are then firmly pressed down and sealed with wax. (The top of the culture vessel and other bungs are also sealed with wax.) The medium is then allowed to cool and as it cools $\mathrm{CO}_{2}$ is drawn in from the attached balloon. (By opening the clips the filters and tube joining $\mathbf{B}$ and $\mathbf{C}$ are filled with $\mathrm{CO}_{2}$.) This takes some hours and the flasks are then incubated overnight to test for sterility. When the apparatus has been assembled $\mathrm{CO}_{2}$ is passed through for at least two hours before the medium flasks are connected to each other and to the inlet pump joint. In connecting up, the tubes are clipped as near to the joints as possible and the joints rapidly connected to allow as little air as possible to get into 
the joints. The balloon $\mathbf{A}$ is attached to flask $\mathbf{B}$. Carbon dioxide is allowed to flow through the apparatus for a further half-hour and the medium is then slowly pumped from flask $\mathrm{C}$ into the culture vessel. Filling the vessel takes an hour or more, and any residual air in the inlet tubing and pump $D$ is pushed out before the advancing medium and flushed out by the $\mathrm{CO}_{2}$ flow before it can oxidize the medium to any extent. Similarly, any air in the circuit $\mathrm{H}$ is driven out by the action of the pump. When the vessel is filled the $\mathrm{CO}_{2}$ flow is left on at about $50 \mathrm{ml} . / \mathrm{min}$., the pumps are stopped and the whole apparatus left overnight to test for sterility. The vessel can then be inoculated through $\mathrm{G}$ and the bacteria allowed to grow as a batch culture to a suitable population density before the medium inflow and the pump $\mathbf{H}$ are started.

To empty the reservoir I the inlet pump is stopped and the tubing below $\mathbf{D}$ closed with a screw clip; otherwise medium is sucked through the pump into the vessel. Flask $L$ is then attached to the tube $K$ and slight suction applied to $L$. By opening the tap on $\mathrm{K}$ liquid is allowed to flow slowly between $\mathrm{I}$ and $\mathrm{L}$. I is emptied slowly as otherwise too great a vacuum is caused in the flask and the medium in the culture vessel froths vigorously as the $\mathrm{CO}_{2}$ flow increases. The flow of liquid from $\mathrm{I}$ to $\mathrm{L}$ is stopped occasionally to allow the pressure in I to build up again. The cottonwool filter below $\mathbf{J}$ stops contamination, if by chance the bunsen valve at $\mathbf{J}$ leaks during this process After I has been emptied the apparatus is left to equilibrate for a few minutes before the clip at $\mathrm{D}$ is opened and the pump again started.

A 2 l. flask was chosen at $C$ partly because when the medium level in $B$ falls the flow of liquid from $\mathrm{B}$ to $\mathrm{C}$ does not always fully coincide with the flow out of $\mathrm{C}$ and the level in $\mathbf{C}$ falls. If during a long run the level in $\mathbf{C}$ falls too much it is brought back to normal by pushing a hypodermic needle attached to a cottonwool filter through a sealed-off rubber tubing projecting from the top of $\mathrm{C}$ (this is not indicated in Fig. 1) after the tubing has been washed with ethanol, and applying gentle suction. The medium flask $B$ is changed when necessary by closing the rubber tubing near the joint above $B$ and rapidly changing the flask for a similar one.

The pumps have had no parts changed during $2 \frac{1}{2}$ to 3 years of experiments, and have only needed regular cleaning and greasing of the gear boxes and bearings; some wear in the stainless-steel piston rods is now becoming apparent though, and these will need changing soon. The copper in the furnace tube is changed occasionally and this can be done during a run when necessary, without getting air into the system. The $\mathrm{CO}_{2}$ cylinder is always changed well before it is empty as the rate of flow of the last of the gas out of the cylinder is uncertain.

\section{PERFORMANCE OF THE APPARATUS}

Since the water bath containing the culture vessel is large and well stirred the temperature of the medium remains very close to $39^{\circ}$, even with the circulating pump $\mathrm{H}$ running. The pump $\mathrm{H}$ runs continually. The refrigerated bath is thermostatically controlled and cooled by a small refrigerator unit (Grant Instruments, Cambridge). The water in the warm baths is covered with mineral oil to cut down evaporation. Otherwise water tends to distil from the hot baths to the cold glycol solution in the refrigerated bath and raises the level in the latter. When this level gets too high it cools the thermostat unit and water condenses on it and this can 
short circuit the motor or thermostat switch. All electrical connexions in the unit have been covered with a plastic film (Holts', 'Damp-Start') to cut down risks of short circuiting. The pumps are Distillers Co. (Great Burgh, Epsom, Surrey) Micro pumps, of the original type. The newer Series II pump is not so suitable because of a number of points in its construction. Aluminium housings have been built over the pump plungers to cut down dust contamination, and a little ethanol is sprayed over the plungers and glands each day. The pump glands need fractionally tightening each day to take up slack due to wear. Occasionally the Teflon gland packing is renewed (after say 6 months' use). The inlet pump will pump a maximum of $175 \mathrm{ml}$./ $\mathrm{hr}$, and the circulating pump is a bigger model set to pump about $650 \mathrm{ml}$./ $\mathrm{hr}$. The calibration curve of the inlet pump varies from one run to another, but is of constant slope. The calibration in the apparatus also differs from the calibration obtained by pumping water from one vessel to another. The rate of pumping is checked during a run by measuring the volume flowing into the refrigerated reservoir. Variations in pumping speed occur during a run at one setting, but the variation is small. Typical results over $200 \mathrm{hr}$ at a setting to give a dilution rate about $0.2 \mathrm{hr}^{-1}$ were $\mathrm{D}=0 \cdot 188,0 \cdot 181,0 \cdot 181,0 \cdot 212,0 \cdot 218,0 \cdot 207,0 \cdot 199,0 \cdot 186,0 \cdot 184$, the volumes pumped being measured at approximately $20-\mathrm{hr}$ intervals.

The flow of carbon dioxide through the culture vessel is $100 \mathrm{ml} . / \mathrm{min}$. This mixed a dye solution flowing into the vessel in about $1 \mathrm{sec}$. The gas stream impinges on the bottom of the vessel and prevents micro-organisms from settling. Foaming in the vessel has never been serious at the gas flow-rates used, the usual result being a layer of large bubbles about $5 \mathrm{~mm}$. thick or less on top of the medium. Evaporation caused by the gas stream is not serious, amounting to not more than about $2 \%$ of the volume in an overnight batch culture. When the gas flow is above $100 \mathrm{ml} . / \mathrm{min}$. then foaming increases, but flow can drop lower than $100 \mathrm{ml} / \mathrm{min}$., without seriously affecting mixing. The circulating pump alone is not sufficient to stir completely the culture if the gas flow should fail entirely, but this has seldom happened.

Provided that contamination is avoided initially there is little chance of subsequent contamination unless a breakage occurs in one of the glass parts. When contamination has occurred it has usually proved impossible to wash it out completely, even if the contaminating organism (usually a micrococcus) has not swamped the normal growth. Some applications of the apparatus are described in the next paper.

The author wishes to thank Mr W. Smith for assistance during this work.

\section{REFERENCES}

Dawson, P. S. S. (1963). A continuous flow culture apparatus. The cyclone column unit. Can. J. Microbiol. 9, 671.

Elsworth, R., Meakin, L. R. P., Pirt, S. J. \& Capeli, G. H. (1956). A 2 litre scale continuous culture apparatus for micro-organisms. J. appl. Bact. 19, 264.

Fox, M. S. \& Szilatrd, L. (1955). A device for growing bacterial populations under steadystate conditions. J. gen. Physiol. 39, 261.

Herbert, D. (1958). In Recent Progress in Microbiology, 7th int. Congr. for Microbiology. Stockholm. 
Hobson, P. N. \& Smiтh, W. (1962). Continuous culture of anaerobic rumen bacteria. J. Physiol. 164, 6 P.

Hobson, P. N. \& Smith, W. (1963). Continuous culture of rumen bacteria. Nature, Lond. 200, 607 .

Monod, J. (1950). La technique de culture continue. Théorie et applications. Annls Inst. Pasteur, Paris, 79, 390.

Novick, A. \& Sziluad, L. (1950). Description of the chemostat. Science, 112, 715. 